\title{
Adverse Drug Events Observed with the Novel Sodium/Glucose Co-Transporter 2 Inhibitor Ipragliflozin for the Treatment of Patients with Type 2 Diabetes Mellitus: A Systematic Review and Meta- analysis of Randomized Studies
}

\author{
Dan Liu · Hao Chen · Fang Song • Mohamad Anis Ahmed • \\ Hongyan $\mathrm{Wu}$
}

Received: July 29, 2020 / Published online: August 26, 2020

(c) The Author(s) 2020

\section{ABSTRACT}

Introduction: Type 2 diabetes mellitus (T2DM) is becoming a major issue worldwide. To effectively control the blood sugar of patients with T2DM, several novel oral hypoglycemic agents (OHAs) are being developed. Sodium/glucose co-transporter 2 (SGLT 2) inhibitors have recently shown beneficial outcomes in patients with T2DM. In this analysis, we aimed to systematically compare the adverse drug events observed with ipragliflozin versus placebo for the treatment of patients with T2DM.

Methods: http://www.ClinicalTrials.gov, the bibliographic database of life science and

Drs. Dan Liu and Hao Chen contributed equally to this article and are the first co-authors.

Digital Features This article is published with digital features to facilitate understanding of the article. You can access the digital features on the article's associated Figshare page. To view digital features for this article, go to https://doi.org/10.6084/m9.figshare.12789212.

D. Liu · H. Chen · F. Song · H. Wu ( $\varangle)$

Department of Endocrinology, The First Affiliated

Hospital of Yangtze University, Jingzhou First

People's Hospital, Jingzhou, Hubei, People's

Republic of China

e-mail: wcls2020720@163.com

M. A. Ahmed

Department of Cardiology, Peking University

People's Hospital, Beijing, People's Republic of

China biomedical information MEDLINE, EMBASE and the Cochrane Central were searched for English publications satisfying the inclusion and exclusion criteria of this study. Adverse drug events were the end points in this analysis. The latest version (5.4) of the RevMan software was used to analyze the data, and risk ratios (RR) with 95\% confidence intervals (CI) were used to represent the data post analysis.

Results: Eight randomized studies with a total of 1519 participants with T2DM were included in this analysis whereby total treatment-emergent adverse events (RR: 1.06, 95\% CI: 0.96-1.16; $P=0.26$ ), including mild (RR: 0.95, 95\% CI: 0.79-1.13; $\mathrm{P}=0.54$ ), moderate (RR: 1.04, 95\% CI: $0.72-1.51 ; P=0.83)$ and severe treatment-emergent adverse events (RR: 0.72, 95\% CI: $0.26-1.96$; $P=0.52$ ), were not significantly different in those patients who were assigned to ipragliflozin versus placebo for the treatment of T2DM. Moreover, drug-related adverse events (RR: 1.04, 95\% CI: 0.69-1.58; $P=0.85$ ), adverse events leading to drug discontinuation (RR: 1.09, 95\% CI: 0.57-2.10; $P=0.79$ ), urinary tract infection (RR: $1.03,95 \%$ CI: $0.60-1.77 ; P=0.91$ ), naso-pharyngitis (RR: 0.54, 95\% CI: $0.19-1.52 ; P=0.25)$, constipation (RR: $1.94,95 \%$ CI: $0.90-4.20 ; P=0.09$ ), dizziness (RR: $0.81,95 \%$ CI: $0.20-3.23 ; P=0.76$ ), gastrointestinal disorders (RR: 0.96, 95\% CI: $0.68-1.36 ; P=0.82$ ) and dehydration (RR: 2.26, 95\% CI: $0.38-13.43 ; P=0.37)$ were also not significantly different. However, genital 
infection (RR: 4.53, 95\% CI: 1.48-13.85; $P=0.008$ ) and hypoglycemia (RR: $1.68,95 \% \mathrm{CI}$ : 1.03-2.74; $P=0.04$ ) rates were significantly higher in the ipragliflozin group.

Conclusions: The current analysis showed ipragliflozin to be associated with significantly higher genital infection rates compared to placebo, whereas no significant difference was observed compared to the other adverse drug events in these patients with T2DM. In addition, hypoglycemia was also not significantly different following sensitivity analysis.

Keywords: Adverse drug events; Genital infection; Hypoglycemia; Ipragliflozin; Type 2 diabetes mellitus

\section{Key Summary Points}

Why carry out this study?

Type 2 diabetes mellitus (T2DM) is becoming a major issue worldwide. To effectively control the blood sugar of patients with T2DM, several novel oral hypoglycemic agents (OHAs) are being developed.

Sodium/glucose co-transporter 2 (SGLT 2) inhibitors have recently shown beneficial outcomes in patients with T2DM.

\section{What was learned from the study?}

In this analysis, we aimed to systematically compare the adverse drug events observed with ipragliflozin versus placebo for the treatment of patients with T2DM.

Ipragliflozin was associated with significantly higher genital infection rates compared to placebo, whereas no significant difference was observed compared to the other adverse drug events in these patients with T2DM.

\section{INTRODUCTION}

Type 2 diabetes mellitus (T2DM) is becoming a major issue worldwide [1]. To effectively control the blood sugar of patients with T2DM, several novel oral hypoglycemic agents (OHAs) are being developed [2]. Sodium/glucose co-transporter 2 (SGLT 2) inhibitors have recently shown beneficial outcomes in the treatment of patients with T2DM [3].

Ipragliflozin, a novel SGLT 2 inhibitor that was jointly developed by Astellas Pharma and Kotobuki Pharmaceuticals, was approved for the treatment of patients with T2DM in January 2014 [4]. Since then, several studies based on Japanese participants have shown beneficial effects of ipragliflozin compared to placebo [5].

The Fukuoka Study of Ipragliflozin (FUSION) showed several positive outcomes related to glycemic control and cardiovascular parameters in Japanese patients who were treated for T2DM [6]. The interim outcome of the ASSIGN-K Study, which was based on Japanese T2DM participants, also showed ipragliflozin to improve glycemic control and weight loss [7]. Nevertheless, even if adverse drug events were also reported in certain studies, they were never systematically assessed.

In this analysis, we aimed to systematically compare the adverse drug events observed with ipragliflozin versus placebo for the treatment of patients with T2DM.

\section{METHODS}

\section{Search Databases}

http://www.ClinicalTrials.gov, the bibliographic database of life science and biomedical information MEDLINE, EMBASE and the Cochrane Central were the electronic databases searched for English publications satisfying the inclusion and exclusion criteria listed below. 


\section{Search Terms}

The following search terms were considered:

- Ipragliflozin and type 2 diabetes mellitus;

- Ipragliflozin and diabetes mellitus;

- Ipragliflozin and adverse drug outcomes;

- Ipragliflozin and T2DM;

- Novel sodium-glucose co-transporter 2 inhibitor and diabetes mellitus.

These terms were searched from the abovementioned online databases with adherence to the PRISMA study guideline [8].

\section{Inclusion Criteria}

Studies were included if:
(a) They were randomized trials comparing ipragliflozin versus placebo for the treat- ment of patients with T2DM;
(b) They reported adverse drug events as their clinical end points;
(c) They reported any follow-up time period.

\section{Exclusion Criteria}

Studies were excluded if:
(a) They were observational studies/meta- analyses/review of literature/case studies/ letters to editors;
(b) They did not compare ipragliflozin versus placebo;
(c) They did not report adverse drug events as their clinical end points;
(d) They were duplicated/repeated studies.

\section{Participants, Outcomes and Follow-up}

All the participants included in this analysis were patients with T2DM who were treated with ipragliflozin or placebo.

The following end points were assessed in this analysis (Table 1):
(a) Total treatment-emergent adverse events (TEAEs);
(b) Mild TEAEs;
(c) Moderate TEAEs;
(d) Severe TEAEs;

(e) Drug-related adverse events;

(f) Adverse events leading to drug discontinuation;

(g) Hypoglycemia;

(h) Urinary tract infection;

(i) Genital infection;

(j) Naso-pharyngitis;

(k) Constipation;

(l) Dizziness;

(m) Gastrointestinal disorders;

(n) Dehydration.

The follow-up time period varied from 2 to 52 weeks as reported in the original studies.

\section{Data Extraction and Quality Assessment}

Important data including the total number of participants assigned to the ipragliflozin and placebo groups, respectively, reported outcomes, follow-up time periods, total number of events representing each end point, baseline features and other main features that were required in this analysis were carefully extracted by the reviewers. Any disagreement that followed was resolved by consensus.

The methodologic quality of the randomized trials was assessed with significant attention given to the criteria recommended by the Cochrane Collaboration [9]. Bias was reported as being of low, moderate or high risk.

\section{Statistical Analysis}

The latest version (5.4) of RevMan software was used to analyze the data. Risk ratios (RRs) and 95\% confidence intervals (CIs) were used to represent the data post analysis.

Heterogeneity was assessed by the $Q$ statistic test. An analysis involving a $P$ value $\leq 0.05$ was considered statistically significant, whereas any $P$ value $>0.05$ was considered statistically insignificant.

Heterogeneity was also assessed by the $I^{2}$ statistic test. The higher the value of $I^{2}$, the higher the heterogeneity was.

The statistical models used included a fixed effect (if $I^{2}<50 \%$ ) and a random effect 
Table 1 End points and follow-up time periods

\begin{tabular}{|c|c|c|c|}
\hline $\begin{array}{l}\text { Randomized } \\
\text { studies }\end{array}$ & Adverse drug events reported & $\begin{array}{l}\text { Follow-up } \\
\text { time period }\end{array}$ & $\begin{array}{l}\text { Type of } \\
\text { participants }\end{array}$ \\
\hline $\begin{array}{l}\text { Fonseca } 2012 \\
\quad[10]\end{array}$ & $\begin{array}{l}\text { TEAEs (mild, moderate, severe), drug-related AEs, drug-related AEs leading } \\
\text { to permanent discontinuation of therapy, hypoglycemia, UTI, GI } \\
\text { disorders }\end{array}$ & 12 weeks & Type 2 DM \\
\hline $\begin{array}{l}\text { Han } 2018 \\
\quad[11]\end{array}$ & $\begin{array}{l}\text { TEAEs (mild, moderate, severe), drug-related AEs, drug-related AEs leading } \\
\text { to permanent discontinuation of therapy, naso-pharyngitis, urticarial, } \\
\text { gastritis, ALT increased, AST increased, hypoglycemia, UTI, genital } \\
\text { infection, dehydration }\end{array}$ & 24 weeks & Type $2 \mathrm{DM}$ \\
\hline $\begin{array}{l}\text { Ishihara } 2016 \\
\quad[12]\end{array}$ & $\begin{array}{l}\text { TEAEs, drug-related AEs, drug-related AEs leading to permanent } \\
\text { discontinuation of therapy, hypoglycemia, UTI, increased blood ketone } \\
\text { bodies, vulvo-vaginal candidiasis, muscle spasm, constipation, rash, } \\
\text { cerebral infarction, dizziness, dehydration }\end{array}$ & 16 weeks & Type $2 \mathrm{DM}$ \\
\hline $\begin{array}{l}\text { Kashiwagi } \\
2015[13]\end{array}$ & $\begin{array}{l}\text { TEAEs, drug-related AEs, drug-related AEs leading to permanent } \\
\text { discontinuation of therapy, hypoglycemia, UTI, genital infection }\end{array}$ & 52 weeks & Type 2 DM \\
\hline Lu $2016[14]$ & $\begin{array}{l}\text { TEAEs (mild, moderate, severe), drug-related AEs, drug-related AEs leading } \\
\text { to permanent discontinuation of therapy, naso-pharyngitis, osteoarthritis, } \\
\text { UTI, dizziness, dysuria, constipation }\end{array}$ & 24 weeks & Type 2 DM \\
\hline $\begin{array}{l}\text { Schwartz } \\
\quad 2011[15]\end{array}$ & TEAEs, GI disorders, constipation, nausea, nervous system disorders & 2 weeks & Type 2 DM \\
\hline $\begin{array}{l}\text { Veltkamp } \\
2012[16]\end{array}$ & $\begin{array}{l}\text { TEAEs (mild, moderate, severe), drug-related AEs, drug-related, naso- } \\
\text { pharyngitis, GI disorders, nausea, dehydration }\end{array}$ & 2 weeks & Type 2 DM \\
\hline $\begin{array}{l}\text { Wilding } 2012 \\
\qquad[17]\end{array}$ & $\begin{array}{l}\text { TEAEs (mild, moderate, severe), drug-related AEs, drug-related AEs leading } \\
\text { to permanent discontinuation of therapy, hypoglycemia, UTI, GI } \\
\text { disorders, urticarial, rash, nervous system disorder, UTI, genital infection }\end{array}$ & 12 weeks & Type 2 DM \\
\hline
\end{tabular}

$\overline{D M}$ diabetes mellitus, TEAEs treatment-emergent adverse events, AEs adverse events, UTI urinary tract infection, $G I$ gastrointestinal

$\left(I^{2}>50 \%\right)$ model depending on the $\mathrm{I}^{2}$ value obtained.

Sensitivity analysis was carried out by an exclusion method.

Publication bias was visually assessed through funnel plots for this analysis with a low study volume.

\section{Compliance with Ethics Guidelines}

This article is based on previously conducted studies and does not contain any studies with human participants or animals performed by any of the authors.

\section{RESULTS}

\section{Search Outcomes}

A total of 1475 publications were obtained from the search databases. After elimination of irrelevant articles, 78 full-text publications were assessed for eligibility.

Further full-text articles were eliminated based on the following criteria: 
- Meta-analyses/literature reviews/observational studies/case studies/letters to editors (7);

- Reported only efficacy outcomes without mentioning adverse drug events (4);

- Did not involve a placebo group (5);

- Reported data that could not be used in this analysis (3);

- Protocol of previous or upcoming trials (5);

- Duplicated studies (46).

Finally, only eight randomized studies [10-17] were confirmed for this analysis, as shown in Fig. 1.

\section{Features of the Randomized Studies and the Included Participants}

Eight randomized studies with a total of 1519 participants with T2DM (1069 were assigned to ipragliflozin and 450 to placebo) were included in this analysis. The time period of patient enrollment varied from the years 2011 to 2017, as shown in Table 2 .

Table 3 lists the baseline features of the participants. The participants had mean age ranging from 53.3 to 65.7 years with a predominance of male participants. HbA1c varied from 7.53 to $8.67 \%$. Duration of diabetes mellitus varied from 4.67 to 14.3 years.

\section{Main Results of This Analysis}

This analysis showed that total treatmentemergent adverse events (RR: 1.06, 95\% CI: 0.96-1.16; $P=0.26$ ), including mild treatmentemergent adverse events (RR: 0.95, 95\% CI: 0.79-1.13; $P=0.54)$, moderate treatmentemergent adverse events (RR: 1.04, 95\% CI: $0.72-1.51 ; \quad P=0.83$ ) and severe treatment-

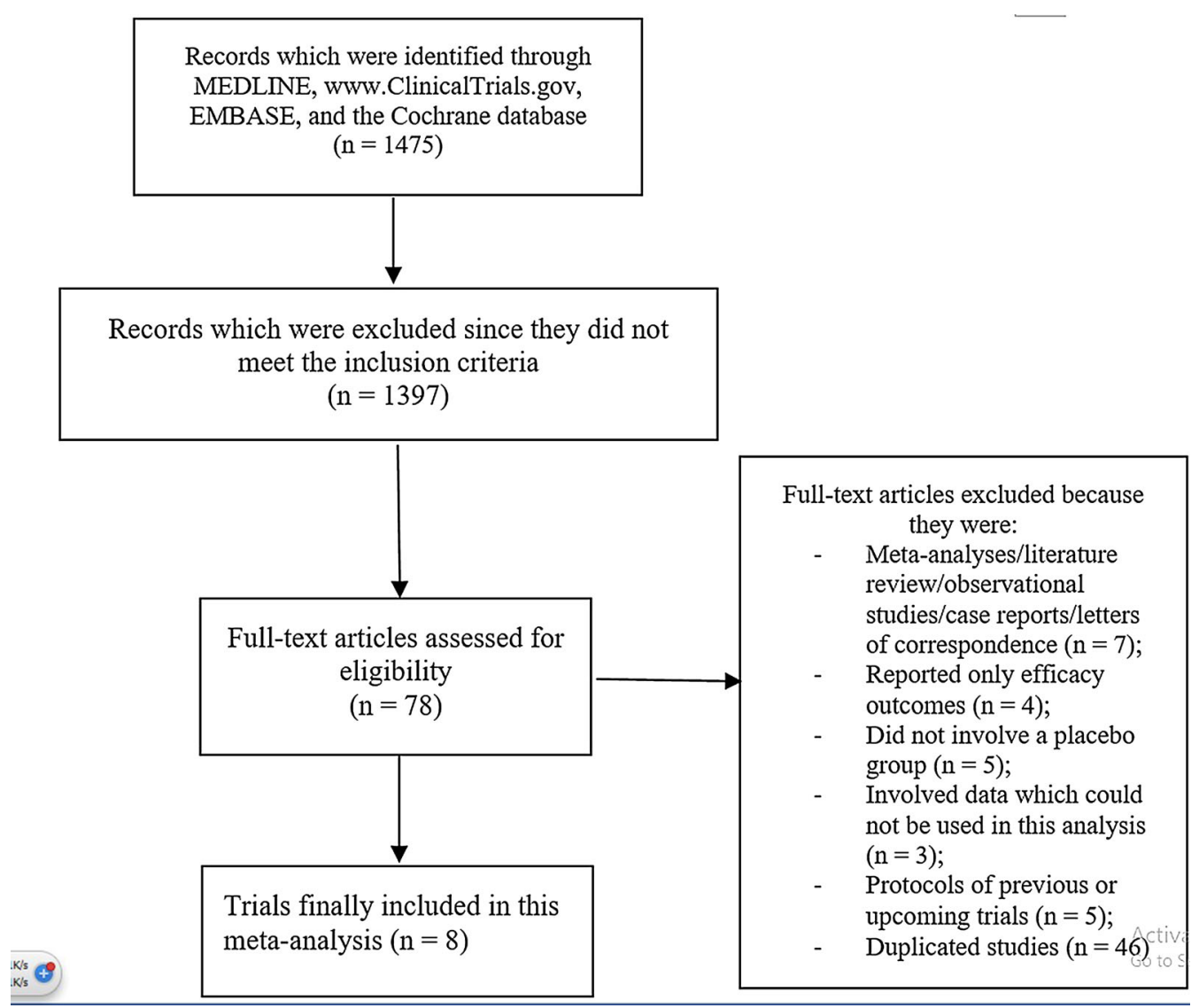

Fig. 1 Flow diagram representing the study selection (PRISMA guideline) 
Table 2 Main features of the randomized studies

\begin{tabular}{llcll}
\hline Studies & $\begin{array}{l}\text { Type of } \\
\text { study }\end{array}$ & $\begin{array}{l}\text { Total no. of participants } \\
\text { assigned to ipragliflozin }(\boldsymbol{n})\end{array}$ & $\begin{array}{l}\text { Total no. of participants } \\
\text { assigned to placebo }(\boldsymbol{n})\end{array}$ & $\begin{array}{l}\text { Time period of } \\
\text { patients' enrollment }\end{array}$ \\
\hline Fonseca 2012 & RT & 273 & 69 & - \\
Han 2018 & RT & 74 & 68 & $2015-2017$ \\
Ishihara 2016 & RT & 175 & 87 & $2014-2015$ \\
Kashiwagi 2015 & RT & 118 & 46 & $2011-2012$ \\
Lu 2016 & RT & 87 & 83 & $2011-2013$ \\
Schwartz 2011 & RT & 48 & 13 & - \\
Veltkamp 2012 & RT & 18 & 18 & 2009 \\
Wilding 2012 & RT & 276 & 66 & - \\
Total no. of & & 1069 & 450 & \\
participants & & & & \\
(n) & & & & \\
\hline
\end{tabular}

$R T$ randomized trials

Table 3 Baseline features of the patients with type 2 diabetes mellitus

\begin{tabular}{llllll}
\hline Studies & $\begin{array}{l}\text { Age (years) } \\
\text { Ipra/placebo }\end{array}$ & $\begin{array}{l}\text { Males (\%) } \\
\text { Ipra/placebo }\end{array}$ & $\begin{array}{l}\text { HbA1c (\%) } \\
\text { Ipra/placebo }\end{array}$ & $\begin{array}{l}\text { Duration of DM (years) } \\
\text { Ipra/placebo }\end{array}$ & $\begin{array}{l}\text { BMI }\left(\mathbf{k g} / \mathbf{m}^{2}\right) \\
\text { Ipra/placebo }\end{array}$ \\
\hline Fonseca 2012 & $53.7 / 53.4$ & $50.9 / 46.4$ & $7.93 / 7.84$ & $4.57 / 4.64$ & $31.2 / 30.9$ \\
Han 2018 & $57.6 / 57.4$ & $50.7 / 48.5$ & $7.90 / 7.92$ & $11.6 / 11.3$ & $25.5 / 26.05$ \\
Ishihara 2016 & $58.7 / 59.2$ & $62.5 / 58.6$ & $8.67 / 8.62$ & $12.6 / 14.3$ & $25.6 / 26.4$ \\
Kashiwagi 2015 & $63.9 / 65.7$ & $78.0 / 78.3$ & $7.53 / 7.55$ & $9.50 / 9.40$ & $25.8 / 24.9$ \\
Schwartz 2011 & $57.9 / 53.3$ & $54.2 / 69.2$ & - & - & $32.5 / 32.4$ \\
Veltkamp 2012 & $58.9 / 55.8$ & $66.7 / 72.2$ & - & - & $31.1 / 31.1$ \\
Wilding 2012 & $57.5 / 57.3$ & $50.4 / 54.5$ & $7.79 / 7.68$ & $6.00 / 5.70$ & $31.7 / 32.0$ \\
\hline
\end{tabular}

HbAlc glycated hemoglobin, DM diabetes mellitus, $B M I$ body mass index, $\mathrm{kg}$ kilogram, $\mathrm{m}^{2}$ square meter, Ipra ipragliflozin group

emergent adverse events (RR: 0.72, 95\% CI: $0.26-1.96 ; P=0.52$ ) were not significantly different in those patients who were assigned to ipragliflozin versus placebo for the treatment of T2DM, as shown in Fig. 2.

Moreover, drug-related adverse events (RR: 1.04, 95\% CI: 0.69-1.58; $P=0.85$ ), adverse events leading to drug discontinuation (RR: 1.09, 95\% CI: $0.57-2.10 ; P=0.79$ ), urinary tract infection (RR: 1.03, 95\% CI: 0.60-1.77; $P=0.91$ ), naso-pharyngitis (RR: $0.54,95 \% \mathrm{CI}$ : 0.19-1.52; $P=0.25$ ), constipation (RR: 1.94, 95\% CI: $0.90-4.20 ; P=0.09$ ), dizziness (RR: 0.81 , 95\% CI: $0.20-3.23 ; P=0.76$ ), gastrointestinal disorders (RR: 0.96, 95\% CI: 0.68-1.36; $P=0.82$ ) and dehydration (RR: $2.26,95 \% \mathrm{CI}$ : $0.38-13.43 ; P=0.37$ ) were also not significantly different, as shown in Figs. 3 and 4. 


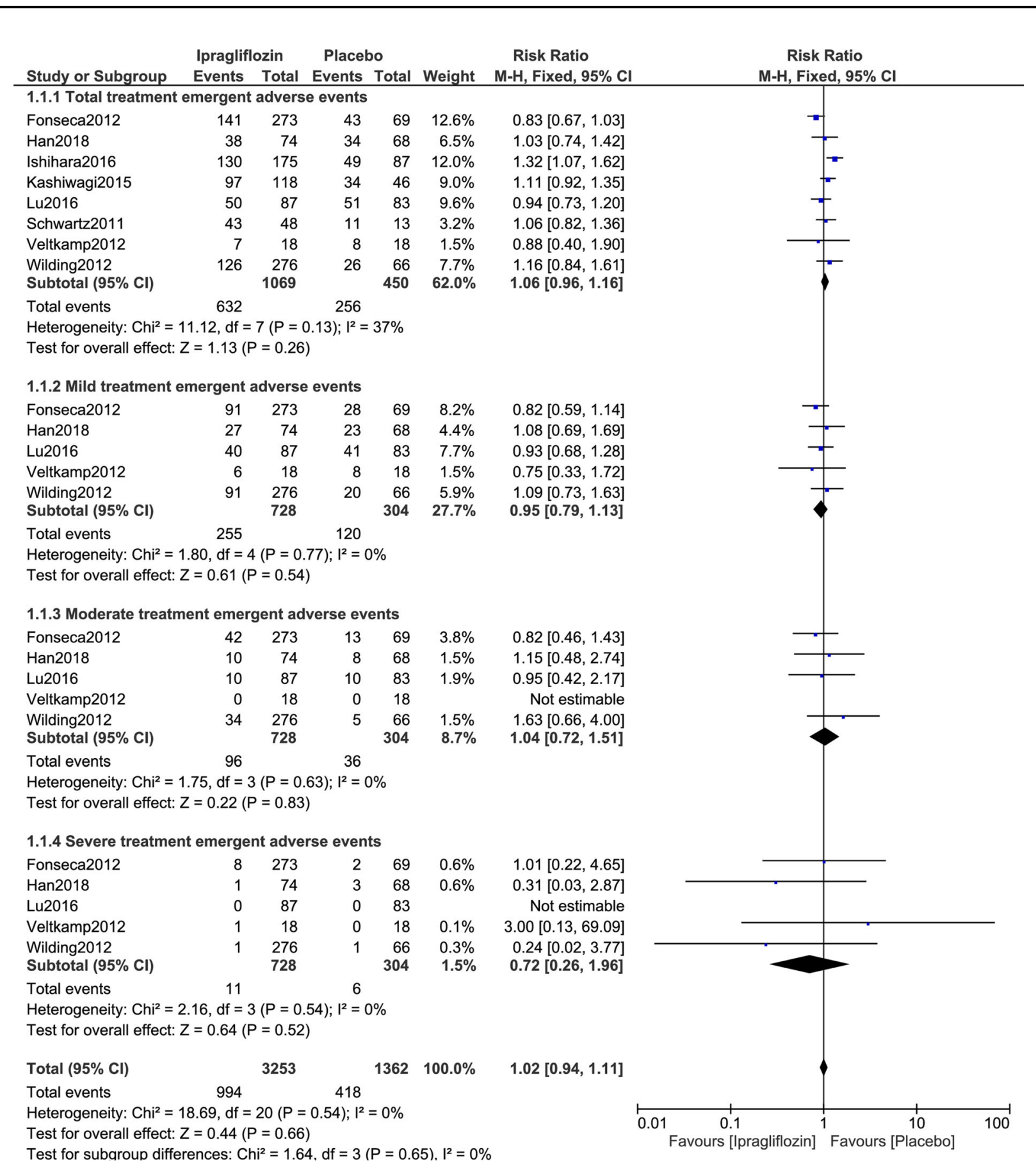

Fig. 2 Adverse drug events observed with ipragliflozin versus placebo for the treatment of patients with type 2 diabetes mellitus

However, genital infection (RR: 4.53, 95\% CI: $1.48-13.85 ; P=0.008)$ and hypoglycemia (RR: $1.68,95 \%$ CI: $1.03-2.74 ; P=0.04$ ) rates were significantly higher in patients who were assigned to ipragliflozin, as shown in Fig. 3.

The results were summarized in Table 4. 


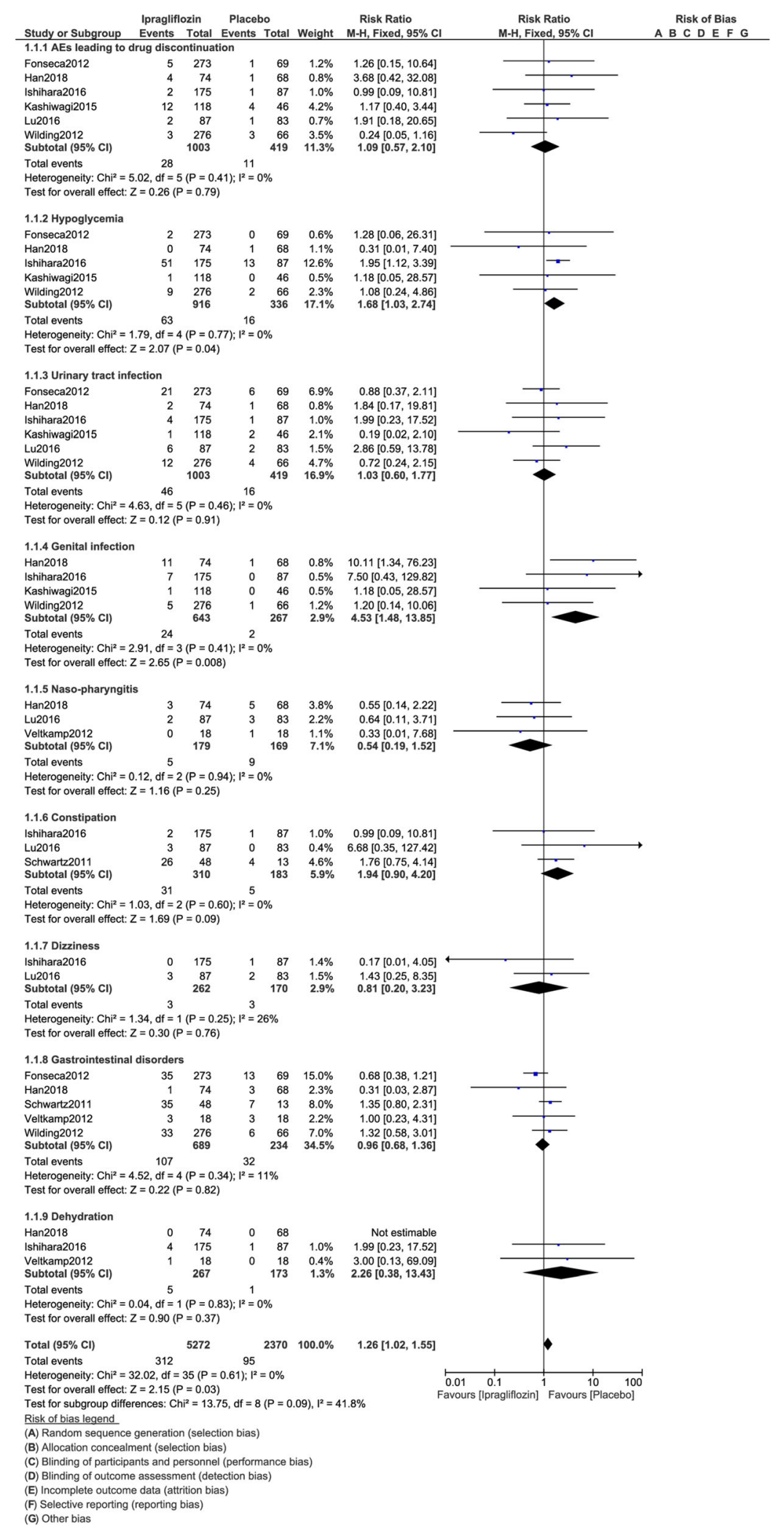


4Fig. 3 Individual adverse drug events observed with ipragliflozin versus placebo for the treatment of patients with type 2 diabetes mellitus

\section{Sensitivity Analysis and Publication Bias}

When sensitivity analysis was carried out, excluding the Fonseca (2012) study (RR: 1.69, 95\% CI: $1.03-2.78 ; P=0.04)$, excluding the Han (2018) study (RR: 1.77, 95\% CI: 1.07-2.93; $P=0.03)$, excluding the Kashiwagi (2015) study (RR: $1.69,95 \%$ CI: $1.03-2.78 ; P=0.04$ ) and excluding the Wilding (2012) study (RR: 1.77, 95\% CI: $1.05-2.98 ; P=0.03$ ) did not show any significant difference compared to the main result, which already showed hypoglycemia to be significantly higher with ipragliflozin. However, excluding the Ishihara (2016) study (RR: $0.92,95 \%$ CI: $0.30-2.85 ; \quad P=0.89$ ) showed hypoglycemia was not significantly higher with ipragliflozin. Sensitivity analysis showed that the main result representing hypoglycemia was influenced by the Ishihara (2016) study and that hypoglycemia might actually not have been significantly higher in those patients who were treated with ipragliflozin. For all the other subgroups, sensitivity analyses showed consistent results throughout.
Publication bias was visually assessed through funnel plots, and low evidence of publication bias was observed across all the randomized studies that assessed the different adverse drug events throughout, as shown in Figs. 5 and 6.

\section{DISCUSSION}

Ipragliflozin, a novel SGLT2 inhibitor, was approved in Japan for the treatment of patients with T2DM in January 2014. Several Japanbased studies have shown this drug to have to be well tolerated by the participants.

In this analysis, where the adverse drug events were compared with ipragliflozin versus placebo, it was found that no significant difference was noted in treatment-emergent adverse events (mild, moderate or severe) and that ipragliflozin was associated with similar rates of urinary tract infection, gastrointestinal disorders, dehydration and other events described in the Results section. However, the genital infection rate was significantly higher with ipragliflozin.

Even though our result showed hypoglycemia to be significantly higher with ipragliflozin, sensitivity analyses showed that this result was highly influenced by the data from

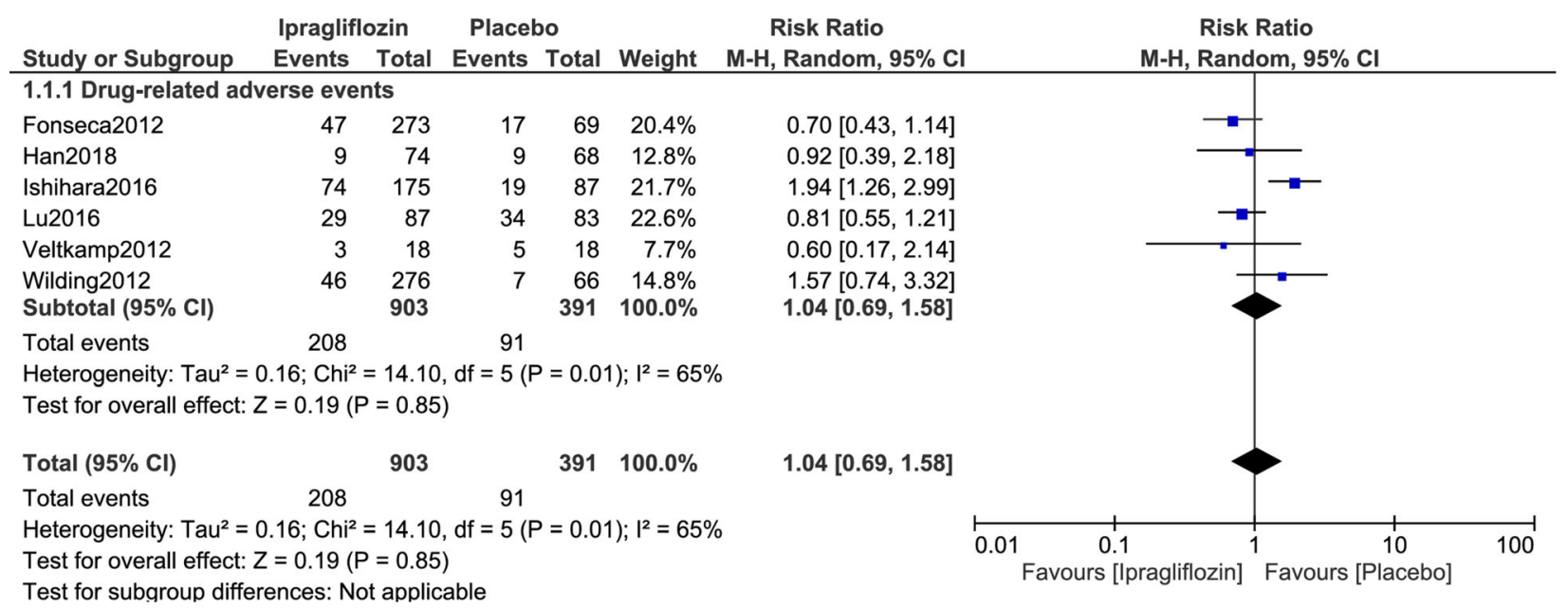

Fig. 4 Drug-related adverse events observed with ipragliflozin versus placebo for the treatment of patients with type 2 diabetes mellitus 
Table 4 Summarized results

\begin{tabular}{lllc}
\hline Adverse drug events assessed & RR with 95\% CI & $\boldsymbol{P}$ value & $\boldsymbol{I}^{\mathbf{2}}$ (\%) \\
\hline Total TEAEs & $1.06[0.96-1.16]$ & 0.26 & 37 \\
Mild TEAEs & $0.95[0.79-1.13]$ & 0.54 & 0 \\
Moderate TEAEs & $1.04[0.72-1.51]$ & 0.83 & 0 \\
Severe TEAEs & $0.72[0.26-1.96]$ & 0.52 & 0 \\
Drug-related adverse events & $1.04[0.69-1.58]$ & 0.85 & 65 \\
AEs leading to drug discontinuation & $1.09[0.57-2.10]$ & 0.79 & 0 \\
Hypoglycemia & $1.68[1.03-2.74]$ & 0.04 & 0 \\
Urinary tract infection & $1.03[0.60-1.77]$ & 0.91 & 0 \\
Genital infection & $4.53[1.48-13.85]$ & 0.008 & 0 \\
Naso-pharyngitis & $0.54[0.19-1.52]$ & 0.25 & 0 \\
Constipation & $1.94[0.90-4.20]$ & 0.09 & 0 \\
Dizziness & $0.81[0.20-3.23]$ & 0.76 & 26 \\
Gastrointestinal disorders & $0.96[0.68-1.36]$ & 0.82 & 11 \\
Dehydration & $2.26[0.38-13.43]$ & 0.37 & 0 \\
\hline
\end{tabular}

$R R$ risk ratios, $C I$ confidence intervals, TEAEs treatment-emergent adverse events, $A E s$ adverse events

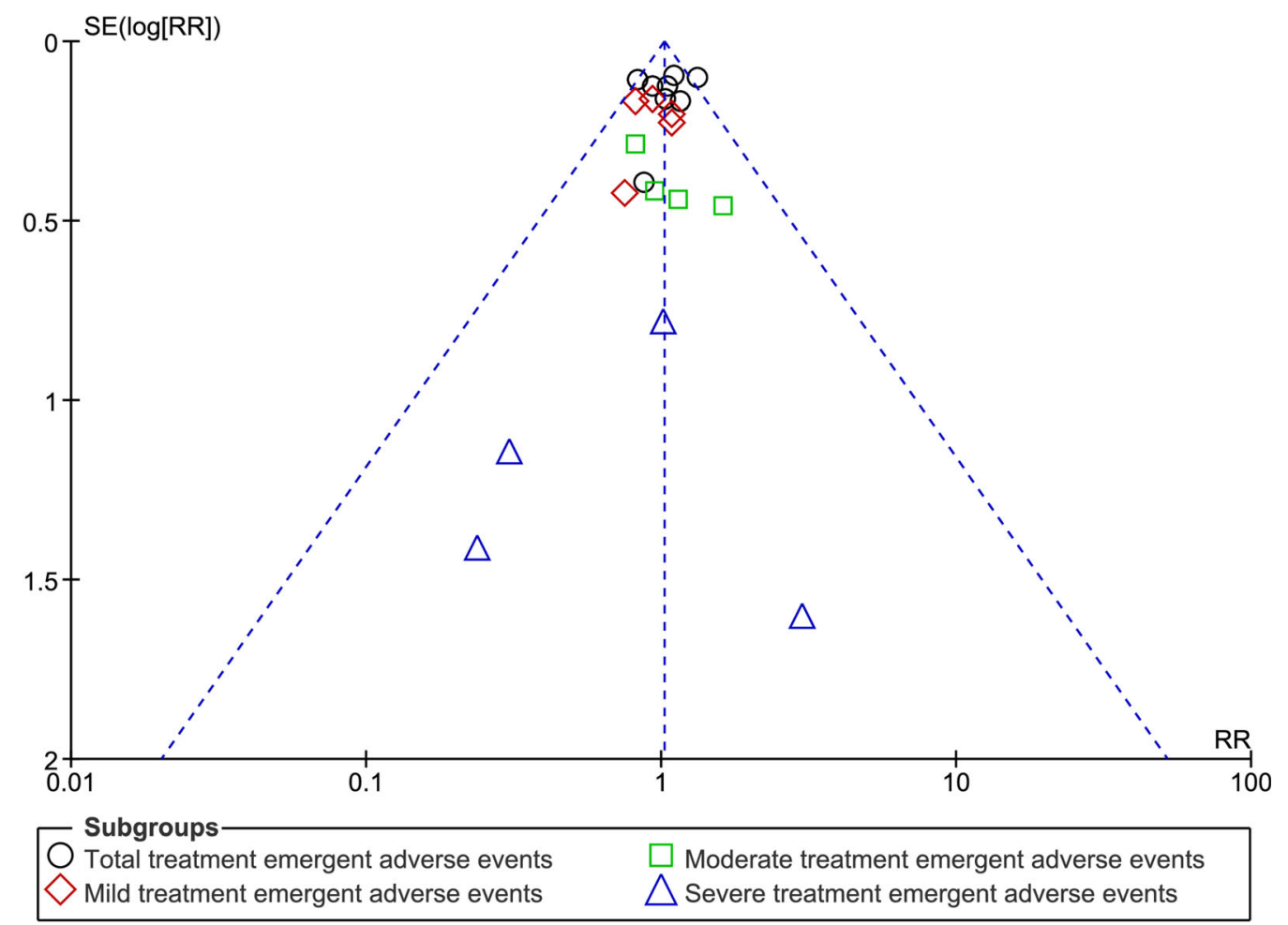

Fig. 5 Funnel plot representing publication bias (A) 


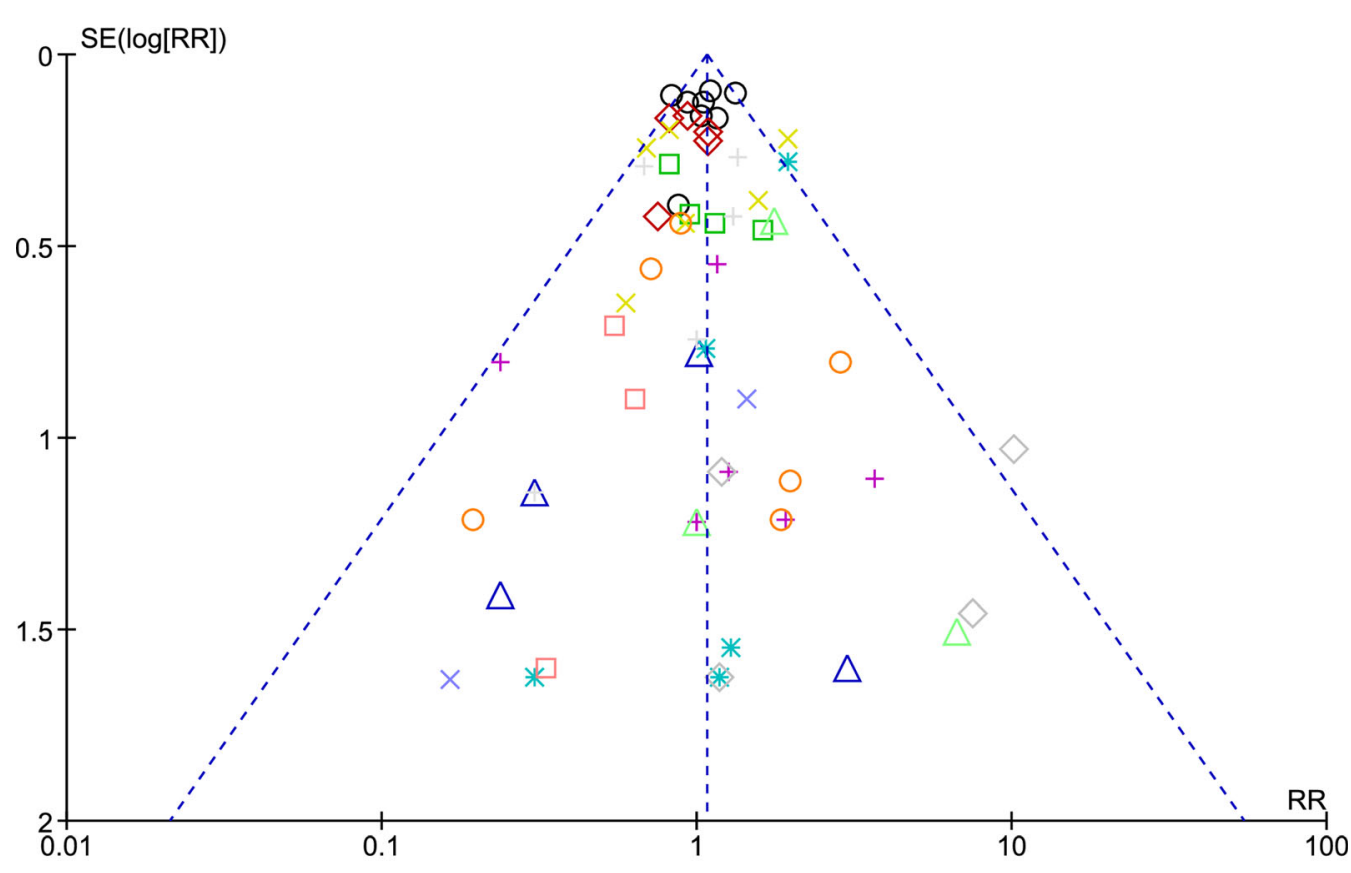

\begin{tabular}{|ll|}
\hline Subgroups & \\
$\bigcirc$ Total treatment emergent adverse events & $\bigcirc$ Urinary tract infection \\
Mild treatment emergent adverse events & Genital infection \\
$\square$ Moderate treatment emergent adverse events & $\square$ Naso-pharyngitis \\
$\triangle$ Severe treatment emergent adverse events & $\triangle$ Constipation \\
$X$ Drug-related adverse events & Dizziness \\
+ AEs leading to drug discontinuation & Gastrointestinal disorders \\
$*$ Hypoglycemia & \\
\hline
\end{tabular}

Fig. 6 Funnel plot representing publication bias (B)

one particular study. Excluding that particular study showed a similar hypoglycemia risk with ipragliflozin versus placebo throughout.

A recently published meta-analysis showed class one evidence that ipragliflozin is effective and safe to use as mono- or add-on therapy to other oral hypoglycemic agents for the treatment of patients with T2DM [18]. It should be noted that the analysis compared the efficacy and safety outcomes, whereas our analysis compared the adverse drug events. Our results showed significantly higher genital infection rates to be associated with the use of ipragliflozin, which makes this the usefulness of this treatment doubtful.

The STELLA-ELDER trial also showed that this drug was well tolerated in patients with T2DM even though a small percentage of the participants had genital infections [19]. Hypoglycemia and genital infections were of a non- serious type. Even during the long term, as shown in the interim results of the STELLALong-Term post-marketing surveillance study, ipragliflozin was still well tolerated and effective in Japanese patients with T2DM without showing any new safety issues [20].

Additionally, even though diabetic ketoacidosis was not assessed in this current paper, some studies have also reported ketoacidosis following the administration of ipragliflozin [21]. Therefore, it is recommended to monitor euglycemic ketoacidosis following the discontinuation of ipragliflozin especially in those patients who do not feel well after discontinuing the drug. Other important adverse outcomes of ipragliflozin could include dehydration and frailty. A study even mentioned that ipragliflozin should be used cautiously in elderly patients because of a higher risk of renal impairment, orthostatic hypotension and dehydration [22]. 


\section{Limitations}

The following limitations were reported in this analysis: the total number of participants might not have been sufficient to reach a robust conclusion. In addition, several studies also included an add-on of metformin or insulin with ipragliflozin, and this could have influenced the results. In addition, the duration of disease was not similar in all the studies. Another limitation could be the fact that the dosage of ipragliflozin was not taken into consideration. Moreover, result of the subgroup assessing for hypoglycemia was highly influenced by data from the Ishihara (2016) study showing a significant increase in hypoglycemia with ipragliflozin. However, sensitivity analysis showed that hypoglycemia was not significantly different with ipragliflozin compared to placebo. Another limitation could be the fact that the cardiovascular outcomes were not assessed in this analysis. Cardiovascular outcomes were not reported in the original trials, and our study was only based on the adverse drug reactions. Finally, even if systematic reviews and meta-analyses showed a high evidence level, sometimes rare and severe adverse events cannot be detected in randomized trials because of shorter follow-up time periods in those trials, and this could be another potential limitation of this analysis.

\section{CONCLUSIONS}

The current analysis showed ipragliflozin to be associated with significantly higher genital infection rates compared with placebo, whereas no significant difference was observed for the other adverse drug events in these patients with T2DM. In addition, hypoglycemia was also not significantly different following sensitivity analysis.

\section{ACKNOWLEDGEMENTS}

Funding. No funding or sponsorship was received for this study or publication of this article.
Authorship. All named authors meet the International Committee of Medical Journal Editors (ICMJE) criteria for authorship for this article, take responsibility for the integrity of the work as a whole, and have given their approval for this version to be published.

Authorship Contributions. DL, HC, FS, MAA and HW were responsible for the conception and design, acquisition of data, analysis and interpretation of data, drafting the initial manuscript and revising it critically for important intellectual content. DL and HC wrote the final draft of this manuscript and are the first co-authors of this paper.

Disclosures. Dan Liu, Hao Chen, Fang Song, Mohamad Anis Ahmed and Hongyan Wu have nothing to disclose.

Compliance with Ethics Guidelines. This article is based on previously conducted studies and does not contain any studies with human participants or animals performed by any of the authors.

Data Availability. All data generated or analyzed during this study are included in this published article. References to the original publications have also been provided and are publicly available in all the search databases including MEDLINE, EMBASE and so on.

Open Access. This article is licensed under a Creative Commons Attribution-NonCommercial 4.0 International License, which permits any non-commercial use, sharing, adaptation, distribution and reproduction in any medium or format, as long as you give appropriate credit to the original author(s) and the source, provide a link to the Creative Commons licence, and indicate if changes were made. The images or other third party material in this article are included in the article's Creative Commons licence, unless indicated otherwise in a credit line to the material. If material is not included in the article's Creative Commons licence and your intended use is not permitted by statutory regulation or exceeds the permitted use, you will need to obtain permission directly from the 
copyright holder. To view a copy of this licence, visit http://creativecommons.org/licenses/bync/4.0/.

\section{REFERENCES}

1. Zheng Y, Ley SH, Hu FB. Global aetiology and epidemiology of type 2 diabetes mellitus and its complications. Nat Rev Endocrinol. 2018;14(2):88-98.

2. Ko S-H, Hur KY, Rhee SY, et al. Antihyperglycemic agent therapy for adult patients with type 2 diabetes mellitus 2017: a position statement of the Korean Diabetes Association. Korean J Intern Med. 2017;32(6):947-58.

3. Dai X, Luo ZC, Zhai L, Zhao WP, Huang F. Adverse drug events associated with low-dose $(10 \mathrm{mg})$ versus high-dose $(25 \mathrm{mg})$ empagliflozinin patients treated for type 2 diabetes mellitus: a systematic review and meta-analysis of randomized controlled trials. Diabetes Ther. 2018;9(2):753-70.

4. Approval of Suglat ${ }^{\circledR}$ Tablets, a Selective SGLT2 Inhibitor for Treatment of Type 2 Diabetes, in Japan. Pharma Inc. 2016. Retrieved 15 Nov 2016.

5. Kashiwagi A, Yoshida S, Nakamura I, et al. Efficacy and safety of ipragliflozin in Japanese patients with type 2 diabetes stratified by body mass index: a subgroup analysis of five randomized clinical trials. J Diabetes Investig. 2016;7(4):544-54.

6. Nomiyama T, Shimono D, Horikawa T, et al. Efficacy and safety of sodium-glucose cotransporter 2 inhibitor ipragliflozin on glycemic controland cardiovascular parameters in Japanese patients with type 2 diabetes mellitus; FukuokaStudy of Ipragliflozin (FUSION). Endocr J. 2018;65(8):859-67.

7. Iizuka T, Iemitsu K, Takihata M, et al. Efficacy and safety of ipragliflozin in japanese patients with type 2 diabetes: interim outcome of the ASSIGN-K study. J Clin Med Res. 2016;8(2):116-25.

8. Liberati A, Altman DG, Tetzlaff J, et al. The PRISMA statement for reporting systematic reviews and meta-analyses of studies that evaluate healthcareinterventions: explanation and elaboration. BMJ. 2009;339:b2700.

9. Higgins JP, et al. Assessing risk of bias in included studies. In: Cochrane handbook for systematic reviews of interventions. Wiley. 2008; pp 187-241.

10. Fonseca VA, Ferrannini E, Wilding JP, et al. Activeand placebo-controlled dose-finding study to assess the efficacy, safety, and tolerabilityof multiple doses of ipragliflozin in patients with type 2 diabetes mellitus. J Diabetes Complications. 2013;27(3):268-73.

11. Han KA, Chon S, Chung CH, Lim S, et al. Efficacy and safety of ipragliflozin as an add-on therapy to sitagliptin and metformin in Koreanpatients with inadequately controlled type 2 diabetes mellitus: a randomized controlled trial. Diabetes Obes Metab. 2018;20(10):2408-15.

12. Ishihara H, Yamaguchi S, Nakao I, Okitsu A, Asahina S. Efficacy and safety of ipragliflozin as add-on therapy to insulin in Japanese patients with type 2diabetes mellitus (IOLITE): a multi-centre, randomized, placebo-controlled, double-blind study. Diabetes Obes Metab. 2016;18(12):1207-16.

13. Kashiwagi A, Takahashi $\mathrm{H}$, Ishikawa $\mathrm{H}$, et al. A randomized, double-blind, placebo-controlled study on long-term efficacy and safety of ipragliflozin treatment in patients with type 2 diabetes mellitus and renal impairment: results of the longterm ASP1941 safety evaluation in patients with type 2 diabetes with renal impairment (LANTERN) study. Diabetes Obes Metab. 2015;17(2):152-60.

14. Lu CH, Min KW, Chuang LM, Kokubo S, Yoshida S, Cha BS. Efficacy, safety, and tolerability of ipragliflozin in Asian patients with type 2 diabetes mellitus and inadequate glycemic control with metformin: results of a phase 3 randomized, placebo-controlled, double-blind, multicenter trial. J Diabetes Investig. 2016;7(3):366-73.

15. Schwartz SL, Akinlade B, Klasen S, Kowalski D, Zhang W, Wilpshaar W. Safety, pharmacokinetic, and pharmacodynamic profiles of ipragliflozin (ASP1941), a novel and selective inhibitor of sodium-dependent glucose co-transporter 2, in patients with type 2 diabetes mellitus. Diabetes Technol Ther. 2011;13(12):1219-27.

16. Veltkamp SA, van Dijk J, Collins C, van Bruijnsvoort M, Kadokura T, Smulders RA. Combination treatment with ipragliflozin and metformin: a randomized, double-blind, placebo-controlled study in patients with type 2 diabetes mellitus. Clin Ther. 2012;34(8):1761-71.

17. Wilding JP, Ferrannini E, Fonseca VA, Wilpshaar W, Dhanjal P, Houzer A. Efficacy and safety of ipragliflozin in patients with type 2 diabetes inadequately controlled on metformin: a dose-finding study. Diabetes Obes Metab. 2013;15(5):403-9.

18. Elgebaly A, Abdelazeim N, Abdelazeim B, El Ashal G, Mattar O, Namous L, Nasreldin N. Tolerability and efficacy of ipragliflozin in the management of inadequately controlled type 2 diabetes mellitus: a systematic review and meta-analysis. Exp Clin Endocrinol Diabetes. 2018. 
19. Terauchi Y, Yokote K, Nakamura I, Sugamori H. Safety of ipragliflozin in elderly Japanese patients with type 2 diabetes mellitus (STELLA-ELDER): interim results of a post-marketing surveillance study. Expert Opin Pharmacother. 2016;17(4): 463-71.

20. Nakamura I, Maegawa H, Tobe K, Tabuchi H, Uno S. Safety and efficacy of ipragliflozin in Japanese patients with type 2 diabetes in real-world clinical practice: interim results of the STELLA-LONG TERM post-marketing surveillance study. Expert Opin Pharmacother. 2018;19(3):189-201.
21. Miyauchi Masaaki, Toyoda Masao, Fukagawa Masafumi. Atypical Ketoacidosis and Protracted Hyperglycosuria after Treatment with Ipragliflozin, an SGLT2 Inhibitor. Intern Med. 2017;56(13): 1673-8.

22. Scheen AJ. Pharmacodynamics, efficacy and safety of sodium-glucose co-transporter type 2 (SGLT2) inhibitors for the treatment of type 2 diabetes mellitus. Drugs. 2015;75(1):33-59. 